\title{
BMJ Open Improving management of type 2 diabetes in South Asian patients: a systematic review of intervention studies
}

\author{
N Bhurji, ${ }^{1}$ J Javer, ${ }^{2}$ D Gasevic, ${ }^{1}$ N A Khan ${ }^{2,3}$
}

To cite: Bhurji N, Javer J, Gasevic D, et al. Improving management of type 2 diabetes in South Asian patients: a systematic review of intervention studies. BMJ Open 2016;6:e008986. doi:10.1136/bmjopen-2015008986

- Prepublication history and additional material is available. To view please visit the journal (http://dx.doi.org/ 10.1136/bmjopen-2015008986).

Received 3 June 2015 Revised 2 September 2015 Accepted 21 October 2015

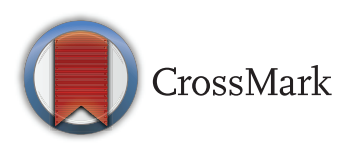

\footnotetext{
${ }^{1}$ College of Medicine and Veterinary Medicine, University of Edinburgh, Edinburgh, UK

${ }^{2}$ Faculty of Medicine, University of British Columbia, Vancouver, British Columbia, Canada

${ }^{3}$ Center for Health Evaluation and Outcome Sciences, Vancouver, British Columbia, Canada
}

Correspondence to Dr NA Khan;

nakhanubc@gmail.com

\section{ABSTRACT}

Objectives: Optimal control of type 2 diabetes is challenging in many patient populations including in South Asian patients. We systematically reviewed studies on the effect of diabetes management interventions targeted at South Asian patients with type 2 diabetes on glycaemic control.

Design: Systematic review of MEDLINE, EMBASE and CINAHL databases for randomised controlled trials (RCTs) and pre-post-test studies ( January 1990 to February 2014). Studies were stratified by where interventions were conducted (South Asia vs Western countries).

Participants: Patients originating from Pakistan, Bangladesh or India with type 2 diabetes.

Primary outcome: Change in glycated haemoglobin (HbA1c). Secondary end points included change in blood pressure, lipid levels, anthropomorphics and knowledge.

Results: 23 studies (15 RCTs) met criteria for analysis with 7 from Western countries $(\mathrm{n}=2532)$ and 16 from South Asia $(n=1081)$. Interventions in Western countries included translated diabetes education, additional clinical care, written materials, visual aids, and bilingual community-based peers and/or health professionals. Interventions conducted in South Asia included yoga, meditation or exercise, community-based peers, health professionals and dietary education (cooking exercises). Among RCTs in India ( 5 trials; $n=390$ ), 4 demonstrated significant reductions in $\mathrm{HbA} 1 \mathrm{c}$ in the intervention group compared with usual care (yoga and exercise interventions). Among the 4 RCTs conducted in Europe $(n=2161)$, only 1 study, an education intervention of 113 patients, reported a significant reduction in $\mathrm{HbA} 1 \mathrm{c}$ with the intervention. Lipids, blood pressure and knowledge improved in both groups with studies from India more often reporting reductions in body mass index and waist circumference.

Conclusions: Overall, there was little improvement in $\mathrm{HbA1c}$ level in diabetes management interventions targeted at South Asians living in Europe compared with usual care, although other outcomes did improve. The smaller studies in India demonstrated significant improvements in glycaemic and other end points. Novel strategies are needed to improve glycaemic control in South Asians living outside of India.

\section{Strengths and limitations of this study}

- To the best of our knowledge, this is the first systematic review of the efficacy of diabetes management (multiple components) interventions focused on South Asian patients.

- Evidence is stratified by location of study, thereby allowing the examination of chronic diabetes management interventions on South Asians living in India and immigrant South Asian patients living in Western countries.

- Included a systematic review of yoga interventions for diabetes management in South Asian patients with diabetes.

- Given the significant heterogeneity in interventions and outcome results, a pooled analysis was not possible.

- Medication adherence was not addressed in almost all of the trials.

\section{INTRODUCTION}

South Asian persons originating from Pakistan, Bangladesh or India experience a $50 \%$ higher risk of developing type 2 diabetes mellitus compared with other populations, irrespective of whether they live in South Asia or Western countries. ${ }^{1}{ }^{2}$ This high global incidence of type 2 diabetes has significant ramifications on this population as type 2 diabetes is associated with a 2-4 times increased risk of myocardial infarction and stroke compared with those without diabetes. In Canada, age-adjusted standardised mortality is also three times higher in South Asian patients with diabetes compared with South Asian patients without diabetes. ${ }^{1}$ Robust clinical trial evidence demonstrates that reducing glycated haemoglobin (HbA1c), blood pressure and fasting blood lipids, using lifestyle modification and diabetes treatment, reduces total mortality by $46 \%$, cardiovascular death by $57 \%$ and non-fatal cardiovascular events by $59 \% .^{3}{ }^{4}$ As such, multiple 
national guideline bodies and diabetes programmes recommend diabetes education and behaviour modification to promote diet, exercise and diabetes medication adherence to achieve these critical reductions in HbAlc, blood pressure and lipids.

Despite guidelines and diabetes programmes to improve diabetes management, diabetes control remains suboptimal in many populations including South Asians. ${ }^{56}$ A study in Canada reports that $55 \%$ of South Asian patients are above recommended HbAlc targets, $36 \%$ above blood pressure targets and $58 \%$ are above lipid-level targets for diabetes. ${ }^{5}$ Further, South Asian patients are $24 \%$ less likely to achieve recommended targets for diabetes than the general population and also less likely to exercise or follow a healthy diet. ${ }^{6} 7$ This large gap in diabetes control in South Asians patients propelled the development of novel and culturally adapted diabetes management strategies in this group. Whether such strategies improve diabetes control in South Asian patients remains unclear. Therefore, we conducted a systematic review of all published randomised controlled trials (RCT) and prospective pre-post-test observational studies evaluating lifestyle or educational interventions compared with usual care or control in South Asian patients with type 2 diabetes on change in HbA1c. We also collected data on change in blood pressure, lipid levels, anthropometric measures and diabetes knowledge. We evaluated potential differences in change in $\mathrm{HbA1c}$ by intervention and region of study (South Asia vs Western countries).

\section{METHODS}

The methods were in accordance with the PRISMA checklist for systematic reviews of intervention trials.

\section{Data sources}

We performed a systematic review of intervention studies for type 2 diabetes management in South Asian patients. With the help of a medical librarian, we reviewed MEDLINE, EMBASE and CINAHL databases for articles published between 1 January 1990 and 1 February 2014. The search terms included India, Pakistan, Bangladesh, South Asian, East Indian, diabetes mellitus (type 2), manage, review, management, intervention, disease management, patient compliance, cultural characteristic, attitudes to health, cultural perception, outcomes, access to care and lifestyle changes (see online supplementary file for the full electronic search strategy). Searches were not limited by language. All articles were combined into a single list, and duplicates were excluded, resulting in 271 abstracts for review (see figure 1).

\section{Study selection}

Two authors reviewed all abstracts (NB and JJ; see figure 1). Studies were included if they were: conducted with adult patients identified as South Asian (originating from India, Pakistan or Bangladesh); randomised control trials or pre-post study designs, included any educational or lifestyle intervention for type 2 diabetes management and if they reported on any one of the outcomes of interest: absolute changes in HbAlc (primary outcome of interest), fasting blood glucose, blood pressure, weight, body mass index (BMI), waist circumference (WC), lipid levels and knowledge. We excluded studies where participants were paediatric populations, or had gestational diabetes, prediabetes or type 1 diabetes. We also excluded reviews, cross-sectional, case-control, case series studies as well as drug efficacy studies (including complementary, eg, Ayurvedic medicines). We did not include studies from other South Asian countries as there was a significant paucity of studies in those groups.

\section{Study quality}

All full-text studies that met the selection criteria were evaluated for bias, and were appraised on the basis of a component approach from the Cochrane Review Groups and Jadad score for reporting RCTs. ${ }^{89}$ These methodological quality measures are listed in table 3. Given the nature of the interventions, blinding of participants and caregivers was not possible. We evaluated separately the subgroup of higher quality RCTs that specified appropriate methods or type of randomisation, described losses to follow-up and intention-to-treat analysis.

\section{Data extraction}

Data extraction was completed in duplicate (NB and JJ). Any discrepancies were discussed and consensus achieved (NB, JJ and NAK). Since the outcomes and interventions of the study were clinically heterogeneous, no quantitative data synthesis was performed. Systematic review of the studies was a priori stratified by study location (South Asian countries vs Western countries) as these healthcare delivery systems, and potentially patient populations, were expected to be significantly different. Interventions were also described by type: education, exercise, dietary or mixed studies (defined as a combination of at least two of the aforementioned intervention types). Change in HbA1c from baseline (delta HbA1c) was the primary outcome for this review and was reported, where data were available, as the difference in delta $\mathrm{HbAlc}$ between the intervention and control groups. Findings from duplicate publications were only included once.

\section{RESULTS}

From a review of 274 articles, 23 studies $(n=3613)$ were included in this final review (figure 1 and table 1 ). Seven studies were conducted in Western countries $(\mathrm{n}=2532 \text {, all based in Europe })^{10-17}$ and 16 studies were conducted in people living in South Asia $(n=1081$, all based in India (indigenous Indians)). ${ }^{18-35}$ There were 15 RCTs $(n=3279)$, with $9(n=789)$ conducted in India.

\section{Patient characteristics}

The average age of patients in the studies ranged from 35 to 66 years with the studies from India more often 


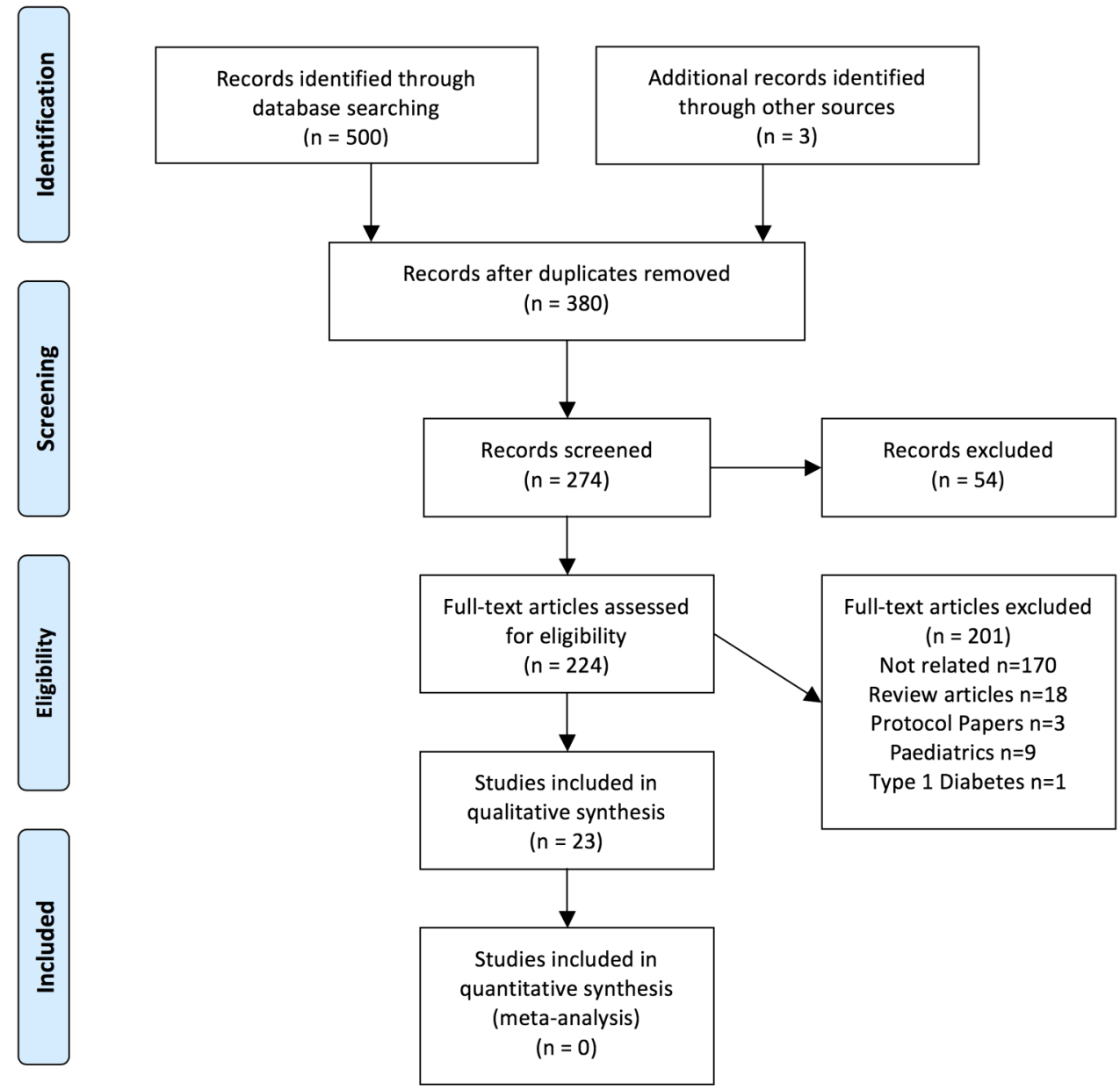

Figure 1 Study flow.

including younger patients (table 2). While approximately half of the patients enrolled in the European-based studies were women, the majority of participants in the India-based studies were men. Mean HbAlc levels at baseline were $\geq 61 \mathrm{mmol} / \mathrm{mol}(7.7 \%)$ and were generally similar between study regions.

\section{Data quality}

Overall, the data quality was modest (table 3). There were five RCTs conducted in India (total $n=390$ ) and four RCTs conducted in Europe (total $n=2161$ ) that measured HbA1c. Only three of the RCTs reported on either type or method of randomisation and quantified loss to follow-up less than $20 \%,{ }^{11} 1221$ and only two trials included information on allocation concealment. ${ }^{12} 21$ Additionally, none of the studies reported on blinding in outcome assessment. Fourteen studies reported loss to follow-up, and two studies had losses greater than $20 \% .^{15} 16$ Only nine studies analysed reported using intention to treat.

\section{Interventions}

There were seven studies of mixed interventions (three from India, four from Europe), eight educational interventions (four from India and three from Europe), four exercise intervention studies (all from India), four yoga intervention studies (all from India) and one study with a diet-based intervention (from India; table 1).

Interventions from studies of South Asians living in Europe Intervention studies with South Asians living in Europe were either mixed interventions or education based. Common features of these interventions included enhanced diabetes education that was culturally specific, the use of bilingual link workers (community members and/or healthcare professionals including nurses or dieticians), as well as the use of written materials and visual aids. Link workers assisted patients with language translation, encouraged patient attendance at clinics, and helped organise educational sessions. One study ${ }^{15}$ included a dietician and podiatrist to provide education using didactic and interactive sessions with visual aids. The other ${ }^{14}$ used peer educators trained by a general practitioner with a special interest in diabetes to provide educational sessions giving advice on exercise, diet, attending appointments, cultural behaviours and the development of diabetes. 
Table 1 Study characteristics by region

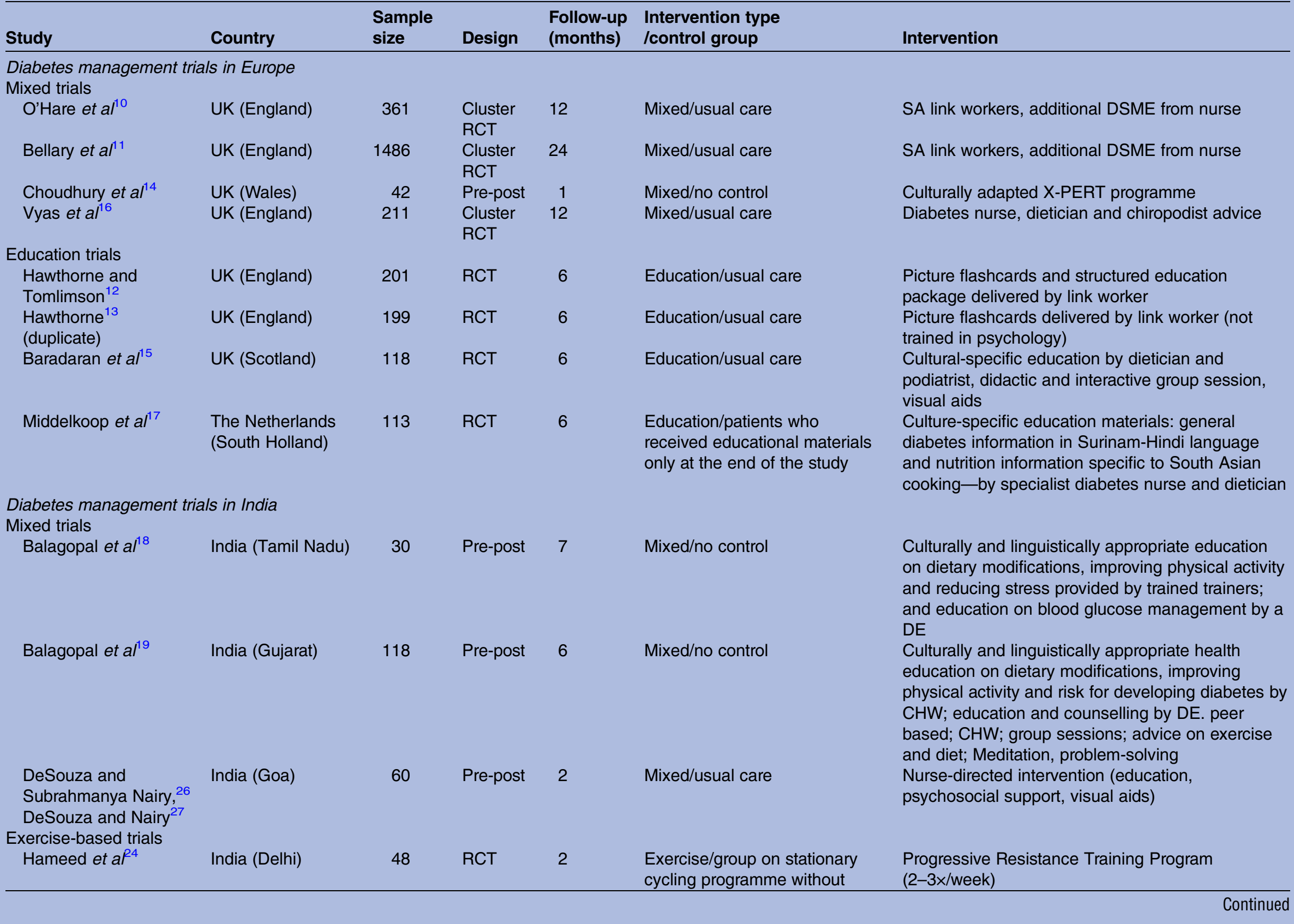

Diabetes management trials in India

\section{Mixed trials}




\begin{tabular}{|c|c|c|c|c|c|c|}
\hline Study & Country & $\begin{array}{l}\text { Sample } \\
\text { size }\end{array}$ & Design & $\begin{array}{l}\text { Follow-up } \\
\text { (months) }\end{array}$ & $\begin{array}{l}\text { Intervention type } \\
\text { /control group }\end{array}$ & Intervention \\
\hline & & & & & $\begin{array}{l}\text { work load and static stretching } \\
\text { exercises }\end{array}$ & \\
\hline Misra et $a f^{5}$ & India (Delhi) & 30 & Pre-post & 3 & Exercise/no control group & $\begin{array}{l}\text { Progressive Resistance Training Program (3×/ } \\
\text { week) }\end{array}$ \\
\hline $\begin{array}{l}\text { Shenoy et } a l^{30} \\
\text { Arora et } a l^{\beta 1}\end{array}$ & India (Punjab) & 30 & $\mathrm{RCT}$ & 4 & $\begin{array}{l}\text { Exercise/participants underwent } \\
\text { no training but continued } \\
\text { medications }\end{array}$ & $\begin{array}{l}\text { Progressive resistance training ( } 2 \text { times per weeks) } \\
\text { and aerobic exercise ( } 3 \text { times a week) }\end{array}$ \\
\hline Shenoy et $a^{\beta 2}$ & India (Punjab) & 40 & RCT & 2 & $\begin{array}{l}\text { Exercise/participants underwent } \\
\text { no training but continued } \\
\text { medications }\end{array}$ & $\begin{array}{l}\text { Aerobic walk using a heart rate monitor and } \\
\text { pedometer to achieve a target of } 150 \text { min per week }\end{array}$ \\
\hline \multicolumn{7}{|c|}{ Yoga-based trials: asanas (body postures) and pranayama (breathing exercises) } \\
\hline Shantakumari et a ${ }^{\text {PO }}$ & India (Kerala) & 100 & RCT & 3 & $\begin{array}{l}\text { Exercise/patients on } \mathrm{OHA} \\
\text { without yogic exercises }\end{array}$ & $\begin{array}{l}\text { OHA and yoga exercises (asanas and pranayama } \\
\text { meditation techniques) }\end{array}$ \\
\hline Vaishali et $a f^{1}$ & India (Karnataka) & 57 & $\mathrm{RCT}$ & 3 & $\begin{array}{l}\text { Exercise/received general } \\
\text { healthy lifestyle and exercise } \\
\text { information }\end{array}$ & $\begin{array}{l}\text { Yogic exercises (asanas) and specially developed } \\
\text { breathing techniques (pranayama) }\end{array}$ \\
\hline Singh et $a^{\beta 4}$ & India (Delhi) & 24 & Pre-post & 1.3 & Exercise & Yoga asanas \\
\hline Madanmohan et a ${ }^{\beta 5}$ & India (Tamil Nadu) & 15 & Pre-post & 1.5 & Exercise & $\begin{array}{l}\text { Yoga (asanas and pranayama) including yogic } \\
\text { counselling and lifestyle modification advice }\end{array}$ \\
\hline \multicolumn{7}{|l|}{ Education trials } \\
\hline Adepu et $a^{2}$ & India (Kerala) & 70 & RCT & 4 & $\begin{array}{l}\text { Education/pharmacist } \\
\text { counselling at study end }\end{array}$ & Patient counselling by pharmacist \\
\hline Ghosh et a f $^{3}$ & $\begin{array}{l}\text { India } \\
\text { (Uttar Pradesh) }\end{array}$ & 22 & $\mathrm{RCT}$ & 1.5 & $\begin{array}{l}\text { Education/pharmacist } \\
\text { counselling and information } \\
\text { leaflets at study end }\end{array}$ & Counselling by pharmacist, information leaflets \\
\hline Malathy et $a^{R^{9}}$ & India (Tamil Nadu) & 207 & $\mathrm{RCT}$ & 3 & $\begin{array}{l}\text { Education/patients received } \\
\text { counselling and information } \\
\text { leaflets at study end }\end{array}$ & Pharmacist counselling and information leaflets \\
\hline Shetty et $a^{\beta 3}$ & India (Tamil Nadu) & 215 & $\mathrm{RCT}$ & 12 & Education/usual care & $\begin{array}{l}\text { SMS messaging on cell phones on diabetes } \\
\text { management education }\end{array}$ \\
\hline \multicolumn{7}{|l|}{ Diet-based trials } \\
\hline Pande et $a^{28}$ & $\begin{array}{l}\text { India } \\
\text { (Maharashtra) }\end{array}$ & 15 & Pre-post & 1 & Diet/no control & Low and low-medium glycaemic load meals \\
\hline
\end{tabular}

Intervention type: education, exercise, dietary or mixed (two or more intervention types).

CBA, controlled before and after experiment; CHW, community health worker; DE, diabetes educator; DSME, diabetes self-management education; OHA, oral hypoglycaemic agent; RCT, randomised controlled trial; SA, South Asian. 


\begin{tabular}{|c|c|c|c|c|c|c|c|c|}
\hline Study author, year & Study population & Recruitment site & $\begin{array}{l}\text { Age } \\
\text { Years } \\
\text { mean }\end{array}$ & $\begin{array}{l}\text { Women } \\
(\%)\end{array}$ & $\begin{array}{l}\text { HbA1c } \\
\text { mmol/ } \\
\text { mol } \\
(\%)\end{array}$ & $\begin{array}{l}\mathrm{BMI} \\
\mathrm{kg} / \mathrm{m}^{2} \text { mean }\end{array}$ & $\begin{array}{l}\mathrm{SBP} \\
\mathrm{mm} \mathrm{Hg} \\
\text { mean }\end{array}$ & $\begin{array}{l}\text { DBP } \mathrm{mm} \mathrm{Hg} \\
\text { mean }\end{array}$ \\
\hline O’Hare, 2004 & $\begin{array}{l}\text { Indian, Pakistani } \\
\text { and Bangladeshi }\end{array}$ & GP practices & 58.9 & 49 & $64(8.0)$ & & 145 & 81 \\
\hline $\begin{array}{l}\text { Bellary and O'Hare, } \\
2008\end{array}$ & $\begin{array}{l}\text { Indian, Pakistani } \\
\text { and Bangladeshi }\end{array}$ & Inner city GP practices & 57.0 & 48 & $63(7.9)$ & 28.5 & 140 & 83 \\
\hline Vyas, 2003 & Pakistani & GP offices & & 46 & & & & \\
\hline \multicolumn{9}{|l|}{ Education trials } \\
\hline Hawthorne, 1997 & Pakistani & $\begin{array}{l}\text { Manchester Diabetes Centre and } 10 \mathrm{GP} \\
\text { practices }\end{array}$ & 53 & 53 & $70(8.5)$ & & & \\
\hline Hawthorne, 2001 & Pakistani & $\begin{array}{l}\text { Manchester Diabetes Centre or diabetes } \\
\text { mini clinics }\end{array}$ & 53.2 & 53 & $68(8.4)$ & & & \\
\hline Baradaran, 2006 & Indian and Pakistani & Daycare centres and GP offices & 58.4 & 49 & & & & \\
\hline Middelkoop, 2001 & $\begin{array}{l}\text { South Asian } \\
\text { Surinamese }\end{array}$ & GP practice and outpatient clinics & 53.3 & 50 & $67(8.3)$ & & & \\
\hline $\begin{array}{l}\text { DeSouza, 2003, } \\
2004\end{array}$ & Indian & Outpatient clinic, private hospitals & $41.0-60.0$ & 28 & & $\begin{array}{l}\text { Obese I: } \\
20 \% \\
\text { C: } 6.7 \%\end{array}$ & $\begin{array}{l}<140 \mathrm{I}: \\
93.3 \% \mathrm{C}: \\
86.7 \%\end{array}$ & \\
\hline \multicolumn{9}{|l|}{ Exercise-based trials } \\
\hline Hameed, 2012 & Indian & University hospital outpatient clinics & 44.7 & 27 & $68(8.4)$ & 27.3 & 130 & 79 \\
\hline Misra, 2008 & Indian & Outpatient and diabetes clinic & 40.8 & 27 & $61(7.7)$ & 24.1 & & \\
\hline Shenoy, 2009 & Indian & University health centre & 53.4 & 47 & $62(7.8)$ & & & \\
\hline Shenoy, 2010 & Indian & Outpatients & 52 & 28 & $57(7.4)$ & 27 & 127 & 86 \\
\hline \multicolumn{9}{|l|}{ Yoga-based trials } \\
\hline Shantakumari, 2013 & South Asian & $\begin{array}{l}\text { Outpatient department of holistic } \\
\text { medicine and university-based diabetes } \\
\text { clinic }\end{array}$ & 45.0 & 49 & & 23.1 & & \\
\hline Vaishali, 2012 & South Asian & Diabetes clinic & 65.8 & 37 & $91(10.5)$ & 27.6 & & \\
\hline Singh, 2004 & Indian & Not specified & $30-60$ & & $75(9.0)$ & & 142 & 86.7 \\
\hline
\end{tabular}




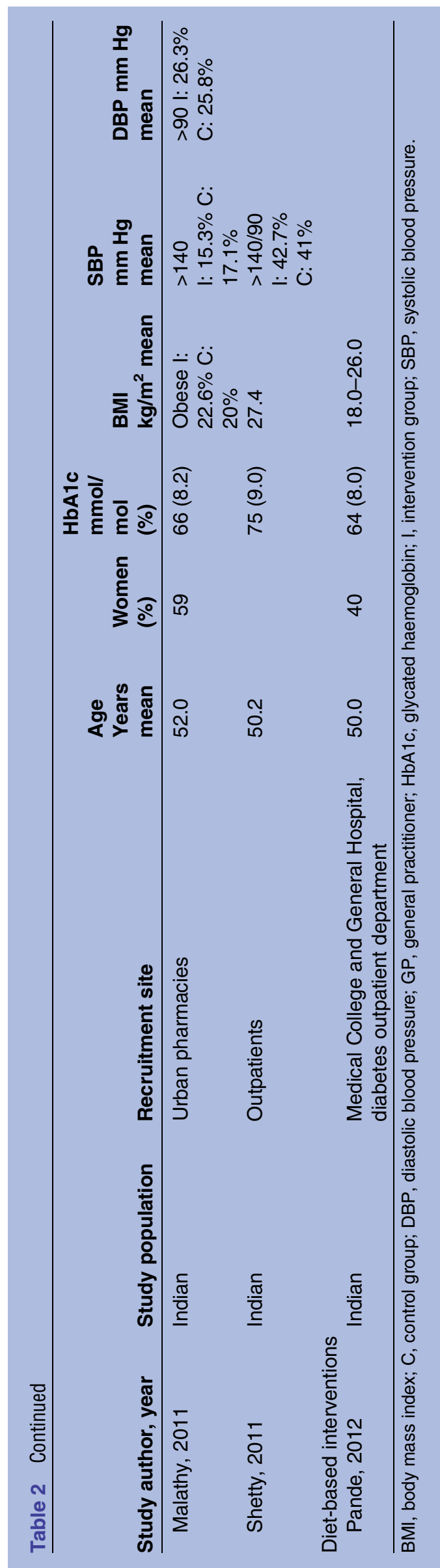

Interventions from studies of indigenous Indians

Studies with indigenous Indians included mixed interventions, exercise, yoga and educational interventions. Common features of these interventions included resistance exercise, and the use of community-based peers and healthcare professionals (pharmacists, nurses) as well as culture-specific dietary education. Three of the four yoga interventions included both asanas (static yoga positions) and pranayama (breathing exercises) and one only included asanas alone. $^{20} \quad 21 \quad 34 \quad 35$ Three exercise studies $^{24} 2530$ used resistance training, while one study included aerobic exercise. ${ }^{30} 31$ The other mixed intervention study from Balagopal et $a l^{18}$ featured education and experiential training on meditation/relaxation techniques, cooking (through demonstrations, recipe competitions and model meals) and exercise (through demonstrations, competitive fun events and dance-based exercise).

\section{Change in HbA1c}

Twelve studies reported HbA1c, while six additional studies included other measures of glycaemia (table 4). Among RCTs conducted in India (5 trials; $n=390$ ), four studies demonstrated significant reductions in HbAlc in the intervention group compared with usual care (yoga and exercise interventions). The yoga interventions were also significant in Northern and Southern India. All exercise interventions were conducted in Northern India, so regional differences could not be ascertained. Among the four RCTs conducted in Europe $(n=2161)$, only one study of an education intervention in 113 patients reported a significant reduction in HbAlc with the intervention, ${ }^{17}$ whereas the remainder found no significant change (mixed and education interventions). Overall, the interventions associated with significant reductions in $\mathrm{HbAlc}$ tended to be based in India, exercise or yoga focused, while the interventions associated with no significant improvement in HbA1c tended to be among the larger trials, South Asian patients living in Europe and focused on enhanced education.

\section{Other end points}

Eight studies reported blood pressure end points; and in four of the five RCT's reporting blood pressure outcomes, interventions were associated with reduced mean systolic (range -0.33 to $-9 \mathrm{~mm} \mathrm{Hg}$ ) or diastolic blood pressure (range -1.91 to $-10.8 \mathrm{~mm} \mathrm{Hg}$ ) compared with usual care. Of the two studies conducted in Europe, both reported significant reductions in either systolic or diastolic blood pressure. Of the six studies of indigenous Indians, four reported significant reductions in blood pressure. Mixed and exercise interventions were associated with blood pressure reduction. None of the education or dietary intervention studies evaluated blood pressure (all conducted in India). Eleven studies evaluated lipid levels with 10 intervention studies demonstrating significant reductions in at least one measured lipid level across all the intervention types (table 4) with two of three studies from Europe and the other eight from India reporting significant 
Table 3 Quality appraisal of trials

\begin{tabular}{|c|c|c|c|c|c|c|c|}
\hline Author, year & Randomised & $\begin{array}{l}\text { Randomisation } \\
\text { method }\end{array}$ & $\begin{array}{l}\text { Type of } \\
\text { randomisation }\end{array}$ & $\begin{array}{l}\text { Allocation } \\
\text { concealment }\end{array}$ & Blinding & $\begin{array}{l}\text { Per cent } \\
\text { Lost to } \\
\text { follow-up }\end{array}$ & ITT \\
\hline Middelkoop & $\mathrm{RCT}$ & Date of birth & NS & NS & None & NS & NS \\
\hline Shenoy, 2009 & RCT & NS & NS & NS & None & 3 & NS \\
\hline Shenoy, 2010 & RCT & NS & NS & NS & None & NS & Yes \\
\hline Shantakumari & $\begin{array}{l}\text { Randomised } \\
\text { parallel study }\end{array}$ & NS & NS & NS & None & NS & NS \\
\hline Vaishali & $\mathrm{RCT}$ & NS & Block & $\begin{array}{l}\text { Sealed opaque } \\
\text { envelopes }\end{array}$ & None & 5.3 & NS \\
\hline Hawthorne & $\mathrm{RCT}$ & $\begin{array}{l}\text { Random number } \\
\text { tables }\end{array}$ & NS & $\begin{array}{l}\text { Sealed } \\
\text { envelopes }\end{array}$ & None & 6.5 & No \\
\hline Adepu & RCT & NS & NS & NS & None & 14.3 & No \\
\hline Baradaran & RCT & NS & Minimisation & NS & None & 37.3 & No \\
\hline Ghosh & RCT & NS & NS & NS & None & Yes & NS \\
\hline Malathy & RCT & NS & NS & NS & None & None & Yes \\
\hline Hameed & RCT & $\begin{array}{l}\text { Random number } \\
\text { tables }\end{array}$ & NS & NS & None & Yes & Yes \\
\hline Shetty & $\mathrm{RCT}$ & $\begin{array}{l}\text { Random number } \\
\text { tables }\end{array}$ & NS & NS & None & NS & No \\
\hline O’Hare & Cluster RCT & None & NS & NS & None & 10.0 & No \\
\hline Bellary & Cluster RCT & Simple & Simple & NS & NS & 10.8 & Yes \\
\hline Vyas & Cluster RCT & NS & By GP practice ${ }^{4}$ & NS & NS & 44 & No \\
\hline $\begin{array}{l}\text { DeSouza, 2003, } \\
2004\end{array}$ & $\begin{array}{l}\text { Pretest } \\
\text { post-test }\end{array}$ & $\begin{array}{l}\text { Convenience } \\
\text { sampling }\end{array}$ & NA & NA & None & NS & Yes \\
\hline Balagopal, 2008 & $\begin{array}{l}\text { Pretest } \\
\text { post-test }\end{array}$ & NA & NA & NA & None & Yes: refusals & Yes \\
\hline Balagopal, 2012 & $\begin{array}{l}\text { Pretest } \\
\text { post-test }\end{array}$ & NA & NA & NA & None & $\begin{array}{l}\text { Yes: } \\
\text { migration } \\
\text { and refusals }\end{array}$ & Yes \\
\hline Mirsa & $\begin{array}{l}\text { Pretest } \\
\text { post-test }\end{array}$ & NA & NA & NA & None & NS & Yes \\
\hline Choudhury & $\begin{array}{l}\text { Pretest } \\
\text { post-test }\end{array}$ & NA & NA & NA & None & 8.9 & No \\
\hline Pande & $\begin{array}{l}\text { Pretest } \\
\text { post-test }\end{array}$ & NA & NA & NA & None & NS & NS \\
\hline Singh & $\begin{array}{l}\text { Pretest } \\
\text { post-test }\end{array}$ & NA & NA & NA & NS & NS & NS \\
\hline $\begin{array}{l}\text { Madanmohan, } \\
2012\end{array}$ & $\begin{array}{l}\text { Pretest } \\
\text { post-test }\end{array}$ & NA & NA & NA & NS & $<1$ & Yes \\
\hline
\end{tabular}

reductions. Six studies among indigenous Indians included BMI or weight measurement with three studies reporting significant reductions (pretest post-test mixed intervention, one yoga and one exercise-based RCT), ${ }^{19} 32$ whereas three others reported no significant reduction or weight increases. All four studies conducted in India that measured WC found significant reductions with the intervention. One European mixed trial measured BMI and WC and identified a significant increase in BMI with the intervention.

Ten studies reported the effect of educational and mixed interventions on knowledge of diabetes, its prevention, management and complications and/or on quality of life (table 5). Among indigenous Indians, the majority of studies targeting diabetes knowledge and related practices as well as patients' quality of life through educational interventions reported significant improvement in the targeted outcomes. Among education studies conducted in Europe targeting knowledge of and practices related to diabetes, education intervention was not consistently associated with the improvement of the outcomes. Specifically, while half of the studies reported an improvement in knowledge of and attitudes and behaviours related to diabetes, the other half either found no such improvement ${ }^{14}$ or the difference in mean change scores between the intervention and control groups did not differ. ${ }^{15}$ Improvement in knowledge was observed in men and women and across literacy levels in one study. ${ }^{13}$

\section{Higher quality randomised studies}

There were three randomised studies with a lower risk of bias $^{11-13} 21$ (two mixed intervention RCTs conducted in 


\begin{tabular}{|c|c|c|c|c|c|c|}
\hline Study & $\begin{array}{l}\mathrm{HbA1c} \\
\mathrm{mmol} / \mathrm{mol}(\%)\end{array}$ & BG (mmol/L) & SBP $(\mathrm{mmHg})$ & DBP (mmHg) & $\begin{array}{l}\text { Lipids } \\
\text { (mmol/L) }\end{array}$ & Anthropometrics \\
\hline \multicolumn{7}{|c|}{ Mixed trials in Europe } \\
\hline O'Hare et al ${ }^{10}$ & $-23(-0.0)(p=0.87)$ & & $-4.6(p=0.035)$ & $-3.4(p=0.003)$ & $\begin{array}{l}\text { TC: }-0.38 \\
(p=0.005)\end{array}$ & \\
\hline Bellary et al ${ }^{11}$ & $-22(-0.2)(p=0.11)$ & & $-0.33(p=0.76)$ & $-1.91(p<0.001)$ & $\begin{array}{l}\text { TC: }+0.03 \\
(p=0.37)\end{array}$ & $\begin{array}{l}\text { BMI: }+0.40(p<0.001) \\
\text { WC: }-0.2(p=0.67)\end{array}$ \\
\hline \multicolumn{7}{|c|}{ Mixed trials in India } \\
\hline $\begin{array}{l}\text { Balagopal } \\
\text { et } a l^{18}\end{array}$ & & $\begin{array}{l}\text { FBG: } \\
-3.34(p=0.03)\end{array}$ & $-3.2(p=0.48)$ & $-5.5(p=0.134)$ & & $\begin{array}{l}\text { BMI: }+0.2(p=0.63) \\
\text { WC: }-4.8 \mathrm{~cm}(p=0.04)\end{array}$ \\
\hline $\begin{array}{l}\text { Balagopal } \\
\text { et } a l^{19}\end{array}$ & 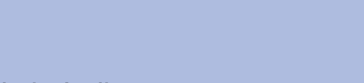 & $\begin{array}{l}\text { FBG: } \\
\text { I -1.06 }(p<0.001)\end{array}$ & I $-6.2(p<0.001)$ & $I-0.17(p<0.001)$ & & $\begin{array}{l}\text { BMI: I }-1.02(p<0.001) \\
\text { WC: I -3.94 cm }(p=0.001)\end{array}$ \\
\hline \multicolumn{7}{|c|}{ Exercise-based trials in India } \\
\hline Hameed et $a l^{24}$ & $-17(-0.6)(p<0.001)$ & & $-4.79(p=0.07)$ & $-1.91(p=0.28)$ & $\begin{array}{l}\text { TC: }-0.07 \\
(p=0.53) \\
\text { TG: }-0.06 \\
(p=0.56) \\
\text { HDL-C:+0.15 } \\
(p=0.004) \\
\text { LDL-C: }-0.19 \\
(p=0.10)\end{array}$ & $\begin{array}{l}\text { Weight }-0.31(p=0.36) \\
\text { WC }(c m):-2.04(p=0.008)\end{array}$ \\
\hline Misra et $a f^{25}$ & $I-18(-0.5)(p<0.001)$ & $\begin{array}{l}\text { FBG: } \\
I-2.7(p<0.001)\end{array}$ & & & $\begin{array}{l}\text { I: TC: }-0.39 \\
(p=0.003) \\
\text { TG: }-0.39 \\
(p<0.001) \\
\text { HDL: }+0.02 \\
(p=0.33) \\
\text { LDL: }-0.09 \\
(p=0.21)\end{array}$ & $\begin{array}{l}\text { I: } B M I:+0.1 \quad(p=0.614) \\
\text { WC: }-1.6(p<0.001)\end{array}$ \\
\hline Shenoy et $a f^{\beta 0}$ & $\begin{array}{l}\text { PRT: }-3(-1.8)(p=0.002) \\
\text { AE: }-9(-1.3)(p=0.002) \\
C:-21(-0.21)(p=N S)\end{array}$ & $\begin{array}{l}\text { PRT: }-3.83(p=0.021) \\
\text { AE: }-1.44(p=0.021) \\
C:-1.66(p=N S)\end{array}$ & $\begin{array}{l}\text { PRT: }-9(p=0.001) \\
\text { AE: }-3(p=N S) \\
C:+2(p=N S)\end{array}$ & $\begin{array}{l}\text { PRT: }-8(p=0.001) \\
\text { AE: }-1(p=N S) \\
C:+1(p=N S)\end{array}$ & & \\
\hline Shenoy et $a \beta^{32}$ & $\begin{array}{l}\text { I: }-15(-0.8)(p=0.0001) \\
C:-23(-0.1) \\
(p=0.242) \\
\text { Difference in post-test I } \\
\text { and C scores } p=0.0023\end{array}$ & $\begin{array}{l}\text { FBG } \\
\text { l: }-4.24(p=0.0001) \\
\text { C: }-0.12(p=0.0007) \\
\text { Difference in post-test I } \\
\text { and C scores } p=0.0001\end{array}$ & $\begin{array}{l}\text { I: }+1.5(p=0.868) \\
C:-3.3(p=0.133) \\
\text { Difference in post-test I } \\
\text { and C scores } p=0.019\end{array}$ & $\begin{array}{l}\text { I: }-10.8(p=0.010) \\
\text { C: }-3.7(p=0.0058) \\
\text { Difference in post-test I } \\
\text { and C scores } p=0.0001\end{array}$ & & $\begin{array}{l}\text { BMI: I: }-1.1(p=0.0001) \\
C:+0.6(p=0.0001) \\
\text { Difference in post-test I and } \\
C \text { scores } p=0.56\end{array}$ \\
\hline \multicolumn{7}{|c|}{ Yoga-based interventions in India } \\
\hline Vaishali et $a f^{1}$ & $-15(-0.8)(p<0.001)$ & $\begin{array}{l}\text { FBG: } \\
-1.58(p<0.001)\end{array}$ & & & $\begin{array}{l}\text { TC: }-0.48 \\
(p<0.001) \\
\text { TG: }-0.25 \\
(p<0.001) \\
\text { HDL:+0.13 } \\
(p<0.001) \\
\text { LDL: }-0.18 \\
(p<0.05)\end{array}$ & \\
\hline
\end{tabular}




\begin{tabular}{|c|c|c|c|c|c|c|}
\hline Study & $\begin{array}{l}\text { HbA1c } \\
\mathrm{mmol} / \mathrm{mol}(\%)\end{array}$ & BG (mmol/L) & SBP (mmHg) & DBP $(\mathrm{mmHg})$ & $\begin{array}{l}\text { Lipids } \\
\text { (mmol/L) }\end{array}$ & Anthropometrics \\
\hline Malathy et $a f^{9}$ & 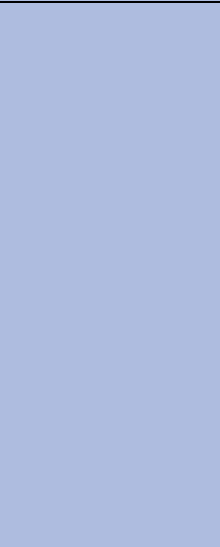 & $\begin{array}{l}\text { Postprandial glucose: } \\
\text { I }-1.81 \\
\text { C }-0.99(p<0.001)\end{array}$ & & & $\begin{array}{l}\text { TC: I-0.53 } \\
(p<0.001) \\
C-0.29 \\
(p<0.05) \\
\text { LDL: I-0.52 } \\
(p<0.001) \\
C-0.29 \\
(p<0.01) \\
\text { HDL: I+0.04 } \\
(p<0.05) \\
C+0.02 \text { (p=NS) } \\
\text { TG I-0.12 } \\
(p<0.001) \\
C-0.08 \\
(p=N S)\end{array}$ & \\
\hline $\begin{array}{l}\text { Diet-based interv } \\
\text { Pande, } 2012\end{array}$ & $\begin{array}{l}\text { tions India } \\
I-14(-0.9) \\
(p<0.001)\end{array}$ & $\begin{array}{l}\text { FBG: } \\
\text { I }-1.99 \\
(p<0.001)\end{array}$ & & & $\begin{array}{l}\text { I TG }-0.90 \\
(p<0.001) \\
\text { TC }-1.01 \\
(p<0.001) \\
\text { HDL+0.18 } \\
(p=0.003) \\
\text { LDL }-0.67 \\
(p=0.009)\end{array}$ & \\
\hline
\end{tabular}

${ }^{*}$ Note that 4 of the studies (Baradaran, Choudhury, Vyas and DeSouza) only included knowledge outcomes and thus were not included in this table (see (table 5)).

The numbers represent the difference in change in parameters between the intervention and control groups if not otherwise stated.

AE, aerobic exercise; BG, blood glucose; BMI, body mass index; C, control group; DBP, diastolic blood pressure; HbA1c, glycated haemoglobin; HDL-C, high-density lipoprotein cholesterol; I,

intervention group; LDL-C, low-density lipoprotein cholesterol; NS, not significant; PRT, progressive resistance training group; SBP, systolic blood pressure; TC, total cholesterol; TG,

triglycerides; WC, waist circumference. 
Table 5 Change in knowledge of diabetes, its prevention, management and complications and on quality of life

Studies based in Europe

Hawthorne and Tomlimson ${ }^{12}$

Hawthorne ${ }^{13}$

Education

Choudhury et $\mathrm{al}^{14}$

Education

Baradaran et $a l^{15}$

Education

Vyas et $a l^{16}$

Mixed

Studies based in India

Balagopal et al ${ }^{18}$

Balagopal et al ${ }^{19}$

Adepu et $a^{2}$
Education Change in per cent of patients correctly answering questions about

A. Knowledge: importance of diet $(I+20 \%, C+6 \%)$, food group value scores $(I+14 \%, C 1.5 \%)$, individual food value scores $(\mathrm{I}+13 \%, \mathrm{C}+3.2 \%$; adjusted difference for $\mathrm{I}$ is $+7.8 \%)$, can manage hyperglycaemia $(\mathrm{I}+64 \%, \mathrm{C}$ $+12 \%)$, knowledge about the purpose of screening $(\mathrm{I}+48 \%$, $\mathrm{C}$ baseline data missing to calculate per cent change), knowledge about diabetes complications $(\mathrm{I}+60 \%, \mathrm{C}+1 \%)$ and knowledge about the role of the chiropodist $(\mathrm{I}+35 \%$, $\mathrm{C}$ baseline data missing to calculate per cent change)

B. Attitudes and behaviours: choosing correct food at wedding $(I+14 \%, C-1.9 \%)$, glucose monitoring $(I+29 \%$, C $0 \%)$, number of people checking glucose more than once a week $(\mathrm{I}+33 \%$, C baseline data missing to calculate per cent change), and keeping records of glucose levels $(I+32 \%, C-7 \%)$; however, an attitude that is hard to refuse food at social events did not change after the intervention $(I+10 \%, C+5 \%)$

Change in scores or per cent of patients correctly answering questions related to diabetes in $\mathrm{M}$ and $\mathrm{W}$ : food knowledge scores (I: $\mathrm{M}+11 \%, \mathrm{~W}+14 \%$; $\mathrm{C}: \mathrm{M}+3 \%, \mathrm{~W}+4 \%$ ), knowledge of eye (I: $\mathrm{M}+55 \%, \mathrm{~W}+63 \%$; $\mathrm{C}$ : $\mathrm{M}$ $-3 \%, W+4 \%)$, heart (I: $M+32 \%, W+54 \% ; C: M-3 \%, W-3 \%)$ and feet diabetes complications (I: $M+47 \%, W$ $+67 \%$; C: $M+9 \%, W+17 \%)$, knowledge of chiropody (I: $M+42 \%, W+32 \%$; C: $M+10 \%, W-5 \%)$, can manage hyperglycaemia (I: $M+68 \%, W+63 \%$; $C: M+9 \%, W+13 \%)$, know why to control glucose $(\mathrm{I}: \mathrm{M}+60 \%, \mathrm{~W}+61 \%$; $\mathrm{C}: \mathrm{M}+3 \%$, W $0 \%$ ), know purpose of annual reviews (I: $\mathrm{M}+66 \%, \mathrm{~W}+33 \%$; $\mathrm{C}: \mathrm{M}+14 \%, \mathrm{~W}-7 \%$ ), check glucose regularly (I: $\mathrm{M}+27 \%, \mathrm{~W}+40 \%$; $\mathrm{C}: \mathrm{M}-29 \%, \mathrm{~W} 0 \%)$ and keep glucose records (I: $\mathrm{M}+36 \%, \mathrm{~W}+28 \%$; C: $\mathrm{M}-8 \%$, $\mathrm{W}-4 \%)$ Change in scores or per cent of patients correctly answering questions related to diabetes in LW and IW: food knowledge scores (LW $+12 \%$, IW $+6 \%$ ), knowledge of eye $(\mathrm{LW}+59 \%$, IW $+30 \%)$, heart (LW $+53 \%$, IW $+50 \%)$, and feet ( $\mathrm{LW}+40 \%$, IW $+45 \%$ ), diabetes complications, knowledge of chiropody ( $\mathrm{LW}+42 \%$, IW $+25 \%)$, can manage hyperglycaemia ( $\mathrm{LW}+53 \%$, IW $+55 \%$ ), know why to control glucose (LW $+71 \%$, IW $+42 \%)$, know purpose of annual checks (LW $+44 \%$, IW $+42 \%)$, check glucose regularly $(\mathrm{LW}+20 \%, \mathrm{IW}+17 \%)$

There was no significant change in days study participants adhered to general and specific diet, recommended exercise or foot care (SDSCA Questionnaire); no control group available.

Difference in mean change in scores between I and C groups for knowledge 1.25 ( $p=0.27)$; attitude towards seriousness $-0.17(p=0.76)$; attitude towards complications $0.68(p=0.38)$ and practice $0.76(p=0.23)$. Per cent improvement in mean scores for knowledge (I intervention, EC, WC) about diabetes $(I+12.5 \%(p=0.04)$; EC $+5 \%(p=0.53)$; $W C+12.2 \%(p=0.19)$, attitudes towards seriousness of diabetes $(I+13.5 \%(p=0.005)$; EC $+16.3 \%(p=0.001) ; W C+12.4 \%(p=0.04))$, attitudes towards diabetes complications $(I+8.1 \%(p=0.05) ; E C$ $+1.5 \%(p=0.40)$; WC $+6 \%(p=0.19))$, and practice related to diabetes $(I+20 \%(p=0.005) ; E C+1.7 \%(p=0.63)$; $W C+25 \%(p=0.007))$

Knowledge awareness and self-management questionnaires validated by Hawthorne. I: 0.09 knowledge; -0.15 self-management; C: 0.02 knowledge; 0.29 self-management (differences in prescores and postscores in the I and $\mathrm{C}$ groups)

NS difference between intervention and control

Knowledge score: $+0.94(\mathrm{p}=0.085)$

Diabetes knowledge score (American Diabetes Association risk test): $+0.47(p<0.001)$

Change in per cent of patients correctly answering questions about knowledge of diabetes definition $(I+22, C$ no change), causes of diabetes $(I+67, C-2)$, methods of glucose measurement $(I+43, C+4)$, diabetes complications (kidney $(I+43, C$ no change), eye $(I+27, C-3)$, frequency of eye examinations $(I+67, C$ no 


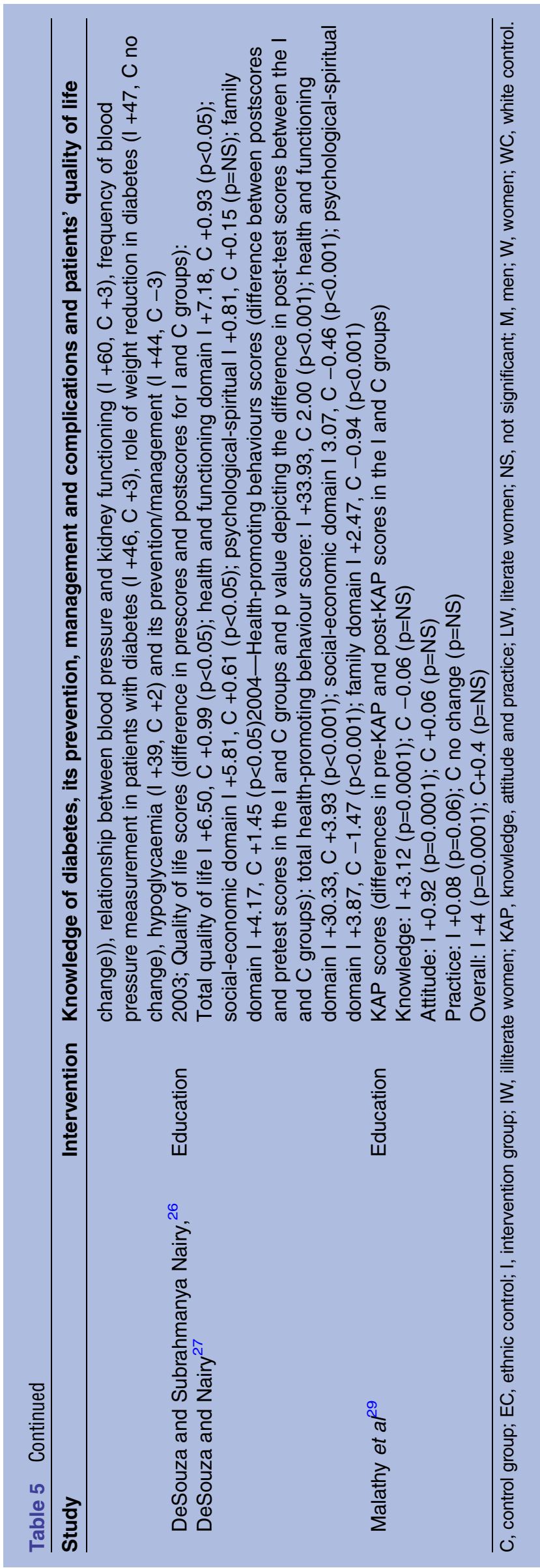

Europe and one yoga-based trial conducted in India). Studies by Bellary $e t a l^{11}$ and Hawthorne ${ }^{12}$ and Hawthorne and Tomlimson, ${ }^{13}$ conducted in Europe (total $\mathrm{n}=1687$ ), used bilingual, bicultural enhanced diabetes education, link workers and visual aids, and they found no significant improvement in HbAlc level with these interventions compared with usual control. In contrast, in the exercise intervention study based in India $(\mathrm{n}=57)$ and led by Vaishali $e t a l^{21}$ there was a significant reduction in HbAlc levels among patients who underwent the intervention compared with their counterparts who did not undergo the exercise intervention.

\section{DISCUSSION}

From the present review, diabetes management interventions targeted at South Asian patients were heterogeneous, yielding variable and limited success in HbAlc reduction. Changes in HbAlc level differed by region and intervention, whereby studies based in India that included exercise or yoga interventions were most successful in reducing HbAlc among individuals with type 2 diabetes. Blood pressure, lipid levels and knowledge were generally improved with various diabetes interventions compared with usual care in European-based and Indian-based studies, whereas BMI did not generally change with the interventions across studies.

The observation that $\mathrm{HbAlc}$ reduction was variable and limited across trials in Europe poses a stark contrast to the findings of culturally adapted diabetes management trials targeted at other ethnic groups studied in the Western world. ${ }^{36-38}$ Meta-analyses of RCTs ascertained that culturally adapted diabetes management programmes are superior to non-culturally adapted programmes with significant improvements $(-17 \mathrm{mmol} /$ mol $(-0.6 \%))$ in HbAlc at 6 months and knowledge scores. ${ }^{36-38}$ Cultural adaptation processes aim to adapt interventions or messages using culture to the intended audience. However, these meta-analyses included largely African-American or Hispanic ethnic groups. Interventions that were face to face, incorporated a peer educator and cognitive reframing techniques were found to be associated with reductions in HbAlc compared with usual care in African-American or Hispanic populations. ${ }^{39}$ In a culturally adapted diabetes programme targeted to a Latin American population, the intervention consisted of use of culturally tailored education, exercise (Latin dance) and dietary change compared with usual care. The cultural adaptations included family involvement, foods common in Latin American countries that could be used in modified Mediterranean diet recipes; and it also incorporated Latin music, language and symbols in meetings and materials. ${ }^{40}$ The intervention group significantly reduced HbAlc $(-19 \mathrm{mmol} / \mathrm{mol}(-0.4 \%))$ at 6 months compared with the usual care group.

Many of the aforementioned culturally adapted techniques were incorporated in the interventions targeted at 
South Asian patients with type 2 diabetes described in this review paper; however, reduction in HbAlc levels was not consistent across studies. We can speculate that the success of the trials from the meta-analysis in reducing HbAlc may lie in the depth to which the interventions were culturally adapted. To illustrate this concept, Resnicow et $a l^{41}$ contrasted surface and deep structural cultural adaptations. Surface structure is based on superficial characteristics of a population including people, language, places and food. Conversely, deep structure involves the integration of the sociocultural concepts, historical context and psychological factors unique to a population. Resnicow et $a l^{t 1}$ states "while adding surface structure to health programming may increase the receptivity of messages, it is deep cultural adaptation that truly fosters salient messaging". In turn, it is possible that the trials from the meta-analysis achieved a greater depth of cultural adaptation compared with the RCTs conducted in Europe and described in this review.

There are several possible explanations underlying the differences in HbAlc changes reported in trials conducted in India compared with trials of patients living in Europe. While the UK studies were larger and mainly adopted an RCT design, the studies in India were generally smaller and eight of the studies employed a pre-post study design (usually a single group) that limits the ability to ascertain causality with the interventions and are associated with some overinflation of effect estimates. ${ }^{42}$ In addition, the variance in results may be explained by differences between the patient populations used in the two different regions. Specifically, trials of South Asians living in India included younger patients and more men than women. It is possible that men may have been more able to engage in physical activity programmes or exerted more control over the diet at home than women. Another possible explanation arises from systemic differences between the two regions. While the UK system is publicly funded, the Indian healthcare system has both private and public funding. Considering privatisation and different levels of socioeconomic position between the regions, there may be significantly less baseline knowledge of diabetes among patients living in India allowing for greater gains with interventions. Additional explanations regarding the differences in outcomes may be rooted in the design of the interventions used in trials. Compared with studies from India, the European studies did not include specific exercise programmes. While it is likely that diabetes selfmanagement education featured in the Europe-based studies advised physical exercise, the trials from India formally integrated yoga and resistance training into their interventions. Studies from India also tended to incorporate experiential-based interventions including meditation training, cooking competitions and dancercise. This raises the possibility that strategies that are immersive and experiential rather than didactic/instructional may lead to better health behaviour change in South Asian groups. Moreover, while all studies from both locations delivered education, advice and training in Indian languages, it is not clear if any deeper cultural adaptations were employed; such adaptations may have been more implicit in the Indian-based interventions. For example, the UK studies featured interventions that were linguistically accessible for the patient population (ie, South Asian link workers, picture flashcards); meanwhile, however, many interventions from India ventured beyond language to include culturally rooted traditions such as specific forms of yoga, meditation and dance. Conversely, South Asian trial participants living in Europe may have been more acculturated compared with those living in India so that cultural adaptation of a diabetes education programme was less effective. Several studies identified significant social stigma attached to having diabetes in South Asian populations that might have negatively influenced their taking part in research studies or fully participating in the research interventions within trials in India and the West. Whether this stigma differs between migrant and indigenous Indians is unknown. Indeed, studies of Western trials demonstrate lower participation of South Asians in clinical studies compared with their non-South Asian counterparts. ${ }^{43}$ Additionally, though all the studies from India reported some statistically significant positive outcomes, it is possible that smaller study effects/publication bias could exist here, and thus small studies from India with negative outcomes may have not been published.

There are several potential reasons underlying the significant intercountry differences in education-based trials, whereby more success in improvement of knowledge of and practices related to diabetes was observed in studies that originated from India. One possible explanation is that the educational interventions from India provided patients with a broader scope of knowledge; that is, information beyond the fundamentals of diabetes self-management. For instance, the intervention conducted by DeSouza and Subrahmanya Nairy ${ }^{26}{ }^{27}$ taught patients about relaxation and stress management as well as the role of family support and participation. Similarly, the programme employed by Balagopal et $a l^{19}$ featured education on meditation and relaxation techniques. Meanwhile, on the basis of the descriptions of UK educational studies, the UK interventions did not appear to venture into these domains. Furthermore, it is also unclear as to whether the success of trials from India assessing patient knowledge was due to something more than just education; specifically, there may exist the inherent ability of the interventions from India to encompass more cultural aspects then we realise. Overall, therefore, the strength of the educational trials from India may have been a blend of encompassing a larger breadth of educational domains and embodying a greater depth of cultural adaptation.

There are several limitations of this systematic review. In terms of the studies selected for the review, the overall quality of the studies was in the low-to-moderate range according to the quality components we studied. 
As such, each study is limited by potential biases. In addition, many of the studies, especially those from India, had smaller sample sizes and interventions between studies varied. Most trials did not report information on blinding of outcome assessment or details of the randomisation, thereby limiting the conclusions of this review. All of the studies conducted in South Asian countries were limited to India, and therefore these findings may not be generalised to other South Asian populations. The lack of studies from other South Asian countries may reflect differences in research support rather than a lower prevalence of diabetes in other South Asian countries. Given the significant heterogeneity in interventions and outcome results, a pooled analysis was not possible. In addition, key components of diabetes management including medication adherence were not addressed in almost all of the trials.

\section{CONCLUSION}

Overall, effectiveness of interventions differed by region. The most successful interventions for reducing HbA1c were more likely to be conducted among South Asians living in India and those interventions included yoga and exercise. While studies from both Western countries and South Asia observed improvements in lipid and blood pressure outcomes, studies from India more often reported reduction in patient BMI, WC and improved diabetes knowledge. Novel interventions are needed to improve glycaemic control of South Asians living in Western countries such as the inclusion of yoga or use of dance to engage participants in improving health behaviours.

Contributors NB and JJ contributed equally as first authors. NAK, NB, JJ and DG were involved with the study design and concept, acquisition of data, analysis and interpretation of data, and preparation and editing of the manuscript.

Funding NAK receives funding from a Michael Smith Foundation for Health Research Career Scientist Award.

\section{Competing interests None declared.}

Provenance and peer review Not commissioned; externally peer reviewed.

Data sharing statement No additional data are available.

Open Access This is an Open Access article distributed in accordance with the Creative Commons Attribution Non Commercial (CC BY-NC 4.0) license, which permits others to distribute, remix, adapt, build upon this work noncommercially, and license their derivative works on different terms, provided the original work is properly cited and the use is non-commercial. See: http:// creativecommons.org/licenses/by-nc/4.0/

\section{REFERENCES}

1. Khan NA, Wang $\mathrm{H}$, Anand $\mathrm{S}$, et al. Ethnicity and sex affect diabetes incidence and outcomes. Diabetes Care 2011;34:96-101.

2. Mohan V, Mathur P, Deepa R, et al. Urban rural differences in prevalence of self-reported diabetes in India-the WHO-ICMR Indian NCD risk factor surveillance. Diabetes Res Clin Pract 2008:80:159-68.

3. Gaede $\mathrm{P}$, Vedel $\mathrm{P}$, Parving $\mathrm{HH}$, et al. Intensified multifactorial intervention in patients with type 2 diabetes mellitus and microalbuminuria: the steno type 2 randomised study. Lancet 1999;353:617-22.
4. Macisaac RJ, Jerums G. Intensive glucose control and cardiovascular outcomes in type 2 diabetes. Heart Lung Circ 2011;20:647-54.

5. Shah BR, Cauch-Dudek K, Anand SS, et al. Absence of disparities in the quality of primary diabetes care for South Asians and Chinese in an urban Canadian setting. Diabetes Care 2012;35:794-6.

6. Gray J, Millett C, Saxena S, et al. Ethnicity and quality of diabetes care in a health system with universal coverage: population-based cross-sectional survey in primary care. J Gen Intern Med 2007;22:1317-20.

7. Liu R, So L, Mohan S, et al. Cardiovascular risk factors in Canadian ethnic populations: results from national surveys. Open Med 2010;4: e143-53.

8. Lundh A, Gøtzsche PC. Recommendations by the Cochrane Review Groups for assessment of the risk of bias in studies. BMC Med Res Methodol 2008;8:22.

9. Moher D, Pham B Jones A, et al. Does quality of reports of randomised trials affect estimates of intervention efficacy reported in meta-analyses? Lancet 1998;352:609-13.

10. O'Hare J, Raymond N, Mughal S, et al. Evaluation of delivery of enhanced diabetes care to patients of South Asian ethnicity: the United Kingdom Asian Diabetes Study (UKADS). Diabet Med 2004;21:1357-65.

11. Bellary S, O'Hare JP, Raymond NT, et al. Enhanced diabetes care to patients of south Asian ethnic origin (the United Kingdom Asian Diabetes Study): a cluster randomised controlled trial. Lancet 2008;371:1769-76.

12. Hawthorne K, Tomlimson S. One-on-one teaching with picturesflashcard health education for British Asians with diabetes. Br J Gen Pract 1997;47:301-4.

13. Hawthorne K. Effect of culturally appropriate health education on glycaemic control and knowledge of diabetes in British Pakistani women with type 2 diabetes mellitus. Health Educ Res Theory Pract 2001;16:373-81.

14. Choudhury S, Brophy S, Fareedi MA, et al. Intervention, recruitment and evaluation challenges in the Bangladeshi community: experience from a peer lead educational course. BMC Med Res Methodol 2008;8:64.

15. Baradaran HR, Knill-Jones RP, Wallia S, et al. A controlled trial of the effectiveness of a diabetes education programme in a multi-ethnic community in Glasgow [ISRCTN28317455]. BMC Public Health 2006;6:134.

16. Vyas A, Haidery AZ, Wiles PG, et al. A pilot randomized trial in primary care to investigate and improve knowledge, awareness and self-management among South Asians with diabetes in Manchester. Diabet Med 2003;20:1022-6.

17. Middelkoop BJC, Geelhoed-Duijvestijn PHLM, van der Wal G. Effectiveness of culture-specific diabetes care for Surinam South Asian patients in the Hague: a randomized controlled trial/ controlled before-and-after study. Diabetes Care 2001;24: 1997-8.

18. Balagopal P, Kamalamma N, Patel TG, et al. A Community-based diabetes prevention and management education program in a rural village in India. Diabetes Care 2008;31:1097-104.

19. Balagopal P, Kamalamma N, Patel TG, et al. A community-based participatory diabetes prevention and management intervention in rural India using community health workers. Diabetes Educ 2012;38:822-34.

20. Shantakumari N, Sequeira S, El deeb R. Effects of a yoga intervention on lipid profiles of diabetes patients with dyslipidemia. Indian Heart J 2013;65:127-31.

21. Vaishali K, Kumar KV, Adhikari P, et al. Effects of yoga-based program on glycosylated hemoglobin level serum lipid profile in community dwelling elderly subjects with chronic type 2 diabetes mellitus - a randomized controlled trial. Phys Occup Ther Geriatr 2012;30:22-30.

22. Adepu R, Rasheed A, Nagavi BG. Effect of patient counseling on quality of life in type-2 diabetes mellitus patients in two selected South Indian community pharmacies: a study. Indian J Pharm Sci 2007;69:519-24.

23. Ghosh S, Rajvanshi A, Kushun S. Assessment the influence of patient counseling on quality of life in type-II diabetes mellitus patients. Int J Pharm Bio Sci 2010;1:1-6.

24. Hameed UA, Manzar D, Raza S, et al. Resistance training leads to clinically meaningful improvements in control of glycemia and muscular strength in untrained middle-aged patients with type 2 diabetes mellitus. N Am J Med Sci 2012;4:336-43.

25. Misra A, Alappan NK, Vikram NK, et al. Effect of supervised progressive resistance-exercise training protocol on insulin 
sensitivity, glycemia, lipids and body composition in Asian Indians with type 2 diabetes. Diabetes Care 2008:31:1282-7.

26. DeSouza M, Subrahmanya Nairy K. Nursing intervention for the quality of life of diabetic adults. Clin Eff Nurs 2003;7:63-72.

27. DeSouza M, Subrahmanya Nairy K. An interventional study on the health promoting behaviours of adults with diabetes. Clin Eff Nurs 2004;8:68-80.

28. Pande A, Krishnamoorthy G, Moulick ND. Hypoglycaemic and hypolipidaemic effects of low GI and medium GL Indian diets in type 2 diabetics for a period of 4 weeks: a prospective study. Int J Food Sci Nutr 2012;63:649-58.

29. Malathy R, Narmadha M, Ramesh S, et al. Effect of a diabetes counseling programme on knowledge, attitude and practice among diabetic patients in Erode district of South India. $J$ Young Pharm 2011;3:65-72.

30. Shenoy S, Arora E, Jaspal S. Effects of progressive resistance training and aerobic exercise on type 2 diabetics in Indian population. Int J Diabetes Metab 2009;17:27-30.

31. Arora E, Shenoy S, Sandhu JS. Effects of resistance training on metabolic profile of adults with type 2 diabetes. Indian J Med Res 2009;129:515-19.

32. Shenoy S, Guglani R, Sandhu JS. Effectiveness of an aerobic walking program using heart rate monitor and pedometer on the parameters of diabetes control in Asian Indians with type 2 diabetes. Prim Care Diabetes 2010;4:41-5.

33. Shetty AS, Chamukuttan S, Nanditha A, et al. Reinforcement of adherence to prescription recommendations in Asian Indian diabetes patients using short message service (SMS) - a pilot study. J Assoc Physicians India 2011;59:711-14.

34. Singh S, Malhotra V, Singh KP, et al. Role of yoga in modifying certain cardiovascular functions in type 2 diabetic patients. $J$ Assoc Physicians India 2004;52:203-6.
35. Madanmohan ABB, Dayanidy G, Sanjay Z, et al. Effect of yoga therapy on reaction time, biochemical parameters and wellness score of peri and post-menopausal diabetic patients. Int $J$ Yoga 2012;5:10-15

36. Hawthorne K, Robles Y, Cannings-John R, et al. Culturally appropriate health education for Type 2 diabetes in ethnic minority groups: a systematic and narrative review of randomized controlled trials. Diabet Med 2010;27:613-23.

37. Hawthorne K, Robles Y, Cannings-John R, et al. Culturally appropriate health education for type 2 diabetes mellitus in ethnic minority groups. Cochrane Database Syst Rev 2008;16:3.

38. Liu JJ, Davidson E, Bhopal RS, et al. Adapting health promotion interventions to meet the needs of ethnic minority groups: mixed-methods evidence synthesis. Health Technology Assessment 2012;16:1-469.

39. Ricci-Cabello I, Ruiz-Pérez I, Rojas-García A, et al. Characteristics and effectiveness of diabetes self-management educational programs targeted to racial/ethnic minority groups: a systematic review, meta-analysis and meta-regression. BMC Endocr Disord 2014; $14: 60$

40. Lorig KR, Ritter PL, González VM. Hispanic chronic disease self-management: a randomized community-based outcome trial. Nurs Res 2003;52:361-9.

41. Resnicow K, Baranowski T, Ahluwalia JS, et al. Cultura sensitivity in public health: defined and demystified. Ethn Dis 1999;9:10-21.

42. Carlson KD, Schmidt F. Impact of experimental design on effect size: findings from the research literature on training. J Appl Psychol 1999;84:851-62.

43. Hussain-Gambles M, Leese B, Atkin $\mathrm{K}$, et al. Involving South Asian patients in clinical trials. Health Technol Assess 2004;8:iii, $1-109$. 